\title{
Dynamic features of liver fibrogenesis and fibrosis resolution in the absence of matrix metalloproteinase-9
}

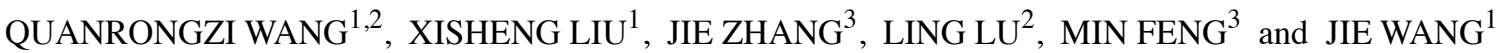 \\ ${ }^{1}$ Department of Radiology, The First Affiliated Hospital of Nanjing Medical University; \\ ${ }^{2}$ Key Laboratory of Living Donor Liver Transplantation, Ministry of Public Health, Nanjing, Jiangsu 210029; \\ ${ }^{3}$ Department of General Surgery, Nanjing Drum Tower Hospital, The Affiliated Hospital of Nanjing \\ Medical University Medical School, Nanjing, Jiangsu 210008, P.R. China
}

Received November 28, 2018; Accepted September 10, 2019

DOI: $10.3892 / \mathrm{mmr} .2019 .10740$

\begin{abstract}
The two-edged effect of matrix metalloproteinase-9 (MMP9) makes it difficult to understand its role in liver fibrogenesis and fibrosis resolution. The present study aimed to investigate the dynamic features of liver fibrogenesis and fibrosis resolution in the absence of MMP9. MMP9-- mice were used to induce liver fibrosis by thioacetamide. The degrees of liver fibrogenesis and fibrosis resolution were designated by the levels of collagen I, III and IV, which were determined via western blotting. Liver injury and the transcriptional levels of MMPs and tissue inhibitor of metalloproteinases (TIMPs) were also determined. It was revealed that, in the absence of MMP9, acute liver injury was attenuated and the expression of collagen was alleviated at the early stage of liver fibrosis, particularly in the first 3 weeks. However, their levels increased to levels as high as those in the control group by week 8 . During liver fibrosis resolution, in the absence of MMP9, the ratio of (MMP9 + MMP13)/TIMP1 and the ratio of (MMP2+ MMP14)/TIMP2 were decreased, and the collagen levels were increased. The present study revealed the dynamic features of liver fibrogenesis and fibrosis resolution in the absence of MMP9. The information obtained here will improve current understanding of the effect that MMP9 has in liver fibrogenesis and fibrosis resolution.
\end{abstract}

Correspondence to: Dr Jie Wang, Department of Radiology, The First Affiliated Hospital of Nanjing Medical University, 300 Guangzhou Road, Gulou, Nanjing, Jiangsu 210029, P.R. China E-mail:wj86202532@163.com

Dr Min Feng, Department of General Surgery, Nanjing Drum Tower Hospital, The Affiliated Hospital of Nanjing Medical University Medical School, 321 Zhongshan Road, Nanjing, Jiangsu 210008, P.R. China

E-mail: fengmindoctor@163.com

Key words: matrix metalloproteinases, tissue inhibitor of metalloproteinases, liver fibrosis, fibrogenesis, fibrosis resolution

\section{Introduction}

Liver fibrosis, the typical response to chronic liver disease and the main factor contributing to the development of liver failure, is regarded as a mixture of fibrogenesis and fibrosis resolution (1-3). The degree of liver fibrosis is largely dependent on the balance between fiber generation and degradation $(4,5)$. Matrix metalloproteinases (MMPs) have been demonstrated to serve a critical role in liver fibrosis by promoting fibrogenesis and fibrosis resolution (6-8).

MMPs are a family of zinc metallo-endopeptidases and their role in promoting liver fibrosis has been investigated extensively (9). The general principle is that MMPs promote the expression of cytokines and chemokines, particularly transforming growth factor $\beta$ (TGF $\beta$ ), by which MMPs activate hepatic stellate cells (HSCs) and promote fibrogenesis (10-12). Previous studies have demonstrated that the depletion of MMP9 or MMP13 suppressed the activation of TGF $\beta$ and the generation of fibrosis in response to acute liver injury $(13,14)$. During liver fibrosis, the generation and degradation of collagen occur simultaneously (2-4). Until now, although numerous MMPs, including MMP1, MMP2, MMP12, MMP13 and MMP14, have been demonstrated to be involved in the regression of liver fibrosis $(4,10,15)$, the underlying molecular mechanism by which MMPs are involved in the degradation of extracellular matrix (ECM) remains unknown. Using a transgene of MMP9, a previous study observed that MMP9 accelerated the resolution of liver fibrosis by neutralizing tissue inhibitor of metalloproteinase-1 (TIMP1) (16). Other studies have indicated that MMPs have a critical role in the apoptosis of activated HSCs, or facilitate the generation of restorative macrophages, which has been proven to promote fibrosis resolution $(17,18)$.

As one of the most important members of the MMP family, MMP9 promotes collagen deposition and degradation. This two-edged effect of MMP9 makes it difficult to understand the precise role of MMP9 in liver fibrosis. The present study aimed to investigate the dynamic features of liver fibrogenesis and fibrosis resolution in the absence of matrix metalloproteinase-9. It was revealed that the absence of MMP9 attenuated liver fibrosis in the early stage of disease, but collagens accumulated in the liver tissues with time and 
reached the same levels as those in the control by 8 weeks. The regression of fibrosis was induced by stopping TAA treatment, and collagen levels were higher in $\mathrm{MMP9}^{-/-}$mice. It has also been suggested that fibronectin peptides generated by limited digestion by MMP9 during liver injury act as local regulatory signals that induce the apoptosis of HSCs, and so prevent chronic fibrogenesis and fibrosis (19).

\section{Materials and methods}

Animal experiments. MMP9-- (FVB) mice from the Model Animal Research Center of Nanjing University (Nanjing, China) were crossed into the B6/C57 background for six generations, and homozygous wild-type (WT) mice were used as controls $(13,14)$. A total of 100 8-weeks old, male mice (20-25 g) were maintained under controlled temperature $\left(22-24^{\circ} \mathrm{C}\right)$ and humidity (50\%) with a 12-h light/dark cycle and were fed standard laboratory chow and water (supplied ad libitum). Animal experimental protocols were approved by the Institutional Animal Care and Research Advisory Committee of Nanjing Drum Tower Hospital (Nanjing, China). The murine model of liver fibrogenesis and fibrosis resolution was prepared as previously described (4) with some modification. Briefly, liver fibrogenesis was induced by repeated intraperitoneal administration of thioacetamide (TAA, $0.1 \mathrm{mg} / \mathrm{g}$ body weight; Sigma-Aldrich; Merck KGaA) every 2 days for 8 weeks. Following all challenges, spontaneous fibrosis resolution was observed for 9 days. Control mice were injected with the same volume of saline.

Serum transaminase and ELISA. Serum was collected by ocular blood extraction at the indicated time points $(0,12,24$, 36 and $48 \mathrm{~h}$ ). Liver injury was estimated according to the increased activity of serum alanine aminotransferase (ALT), which was measured in a clinical biochemical laboratory of Nanjing Drum Tower Hospital. The levels of tumor necrosis factor- $\alpha$ (TNF- $\alpha$ ), interleukin-1 $\beta$ (IL-1 $\beta$ ), IL-10 and TGF $\beta$ in the liver homogenate were determined using commercial ELISA kits (R\&D Systems, Inc.) according to the manufacturer's protocol. All samples and standards were measured in duplicate.

Western blot analysis. The liver samples were prepared as previously described (20). Equal quantities (30 $\mu \mathrm{g})$ of protein extracted from liver tissues were run on 10\% SDS-PAGE gels, followed by electrotransfer onto a polyvinylidene fluoride membrane. The membrane was cut into three for and blocked with $5 \%$ skimmed milk for $2 \mathrm{~h}$ at room temperature and then incubated overnight with primary antibodies at $4^{\circ} \mathrm{C}$. Primary antibodies targeting collagen-I (ab34710, 1:1,000), collagen-III (ab7778, 1:1,000), collagen-IV (ab6586, 1:1,000) and GAPDH (ab181602, 1:2,000) were purchased from Abcam. Horseradish peroxidase-conjugated secondary antibodies (sc-2004, 1:500, Santa Cruz Biotechnology, Inc.) were used $1 \mathrm{~h}$ at room temperature prior to detection with Super Signal West Femto Chemiluminescent substrate (Pierce; Thermo Fisher Scientific, Inc.). The protein band intensities in the western blot analysis were quantified using Image Quant software (version 5.2; GE Healthcare Life Sciences.).

Reverse transcription-quantitative PCR (RT-qPCR) analysis. Total RNA was extracted from liver tissue using TRIzol ${ }^{\circledR}$ (Life
Technologies; Thermo Fisher Scientific, Inc.). The analysis was performed as described previously (20). Briefly, reverse transcription was performed with random primers and the RNAPCR kit (Takara Biotechnology Co., Ltd.). RT-qPCR was conducted according to the manufacturer's instructions using SYBR Premix Ex Taq (Takara Biotechnology Co., Ltd.). Amplifications were performed in a final volume of $20 \mathrm{ml}$ containing $2 \mathrm{ml}$ of cDNA. The reactions were run on the StepOnePlus Real-Time PCR System (Applied Biosystems; Thermo Fisher Scientific, Inc.) using the following program: $95^{\circ} \mathrm{C}$ for $10 \mathrm{~min}$ for the holding stage and 40 cycles of $95^{\circ} \mathrm{C}$ for $15 \mathrm{sec}$ and $60^{\circ} \mathrm{C}$ for $1 \mathrm{~min}$. The expression levels of the target genes were normalized to the housekeeping gene GAPDH. The final result of gene transcription was calculated as $2^{\text {(Ct GAPDH-2Ct Gene) }}(21)$. Analyses were performed using the StepOne Software 2.0 (Applied Biosystems).

The primer sequences used for PCR amplification of the mouse genes were as follows: MMP9, forward 5'-CGTGTC TGGAGATTCGACTTGA-3' and reverse 5'-TGGAAGATG TCGTGTGAGTTCC-3'; MMP13, forward 5'-CCTTCTGGT CTTCTGGCACAC-3', reverse, 5'-GGCTGGGTCGTCACA CTTCTCTGG-3'; MMP2, forward, 5'-CAACGGTCGGGA ATACAGCAG-3' and reverse 5'-CCAGGAAAGTGAAGG GGAAGA-3'; MMP14, forward 5'-ATCTCACAGCTCGGT GTGTGTTCA-3' and reverse 5'-AAGGTCAGAGGGTCT TGCCTTCAA-3'; TIMP1, forward 5'-GCATGGACATTT ATTCTCCACTGT-3' and reverse 5'-TCTCTAGGAGCC CGATCTG-3'; TIMP2, forward 5'-GCCAAAGCAGTGAGC GAGAAG-3' and reverse 5'-GGGGAGGAGATGTAGCAA GGG-3'; GAPDH, forward 5'-AACTTTGGCATTGTGGAA GG-3' and reverse 5'-ACACATTGGGGGTAGGAACA-3'.

Histological examination. The liver tissues were collected at definite times (1, 5 and 9 days after TAA withdrawal) and fixed in $4 \%$ formalin and subsequently embedded in paraffin. The sections were cut into 5-mm slices and were deparaffinized and rehydrated. The slides were incubated with hematoxylin ( 5 min, room temperature) followed by rinsing with water and quick dips into acid ethanol to destain. For eosin staining, the slides were incubated with eosin ( $1 \mathrm{~min}$, room temperature) followed by $95,100 \%$ ethanol and xylene. For Sirius Red staining, the sections were deparaffinized and then stained by Sirius Red (10-15 min, room temperature). The slides were evaluated using light microscopy, as previously described (4).

Statistical analysis. All data are expressed as the mean \pm standard deviation. The differences between two groups were analyzed using two-tailed Student's t-test. Differences between multiple groups were tested with analysis of variance. Statistics and graphs were generated using GraphPad Prism 5.0 (GraphPad Software Inc.). $\mathrm{P}<0.05$ was considered to indicate a statistically significant difference.

\section{Results}

Attenuation of acute liver injury and liver fibrogenesis in the absence of MMP9. Inflammation has been revealed to induce the progression of liver fibrosis (2). In the present study, the role of MMP9 in acute liver injury was investigated. All animals were subjected to the challenge course presented in Fig. 1A. 
A
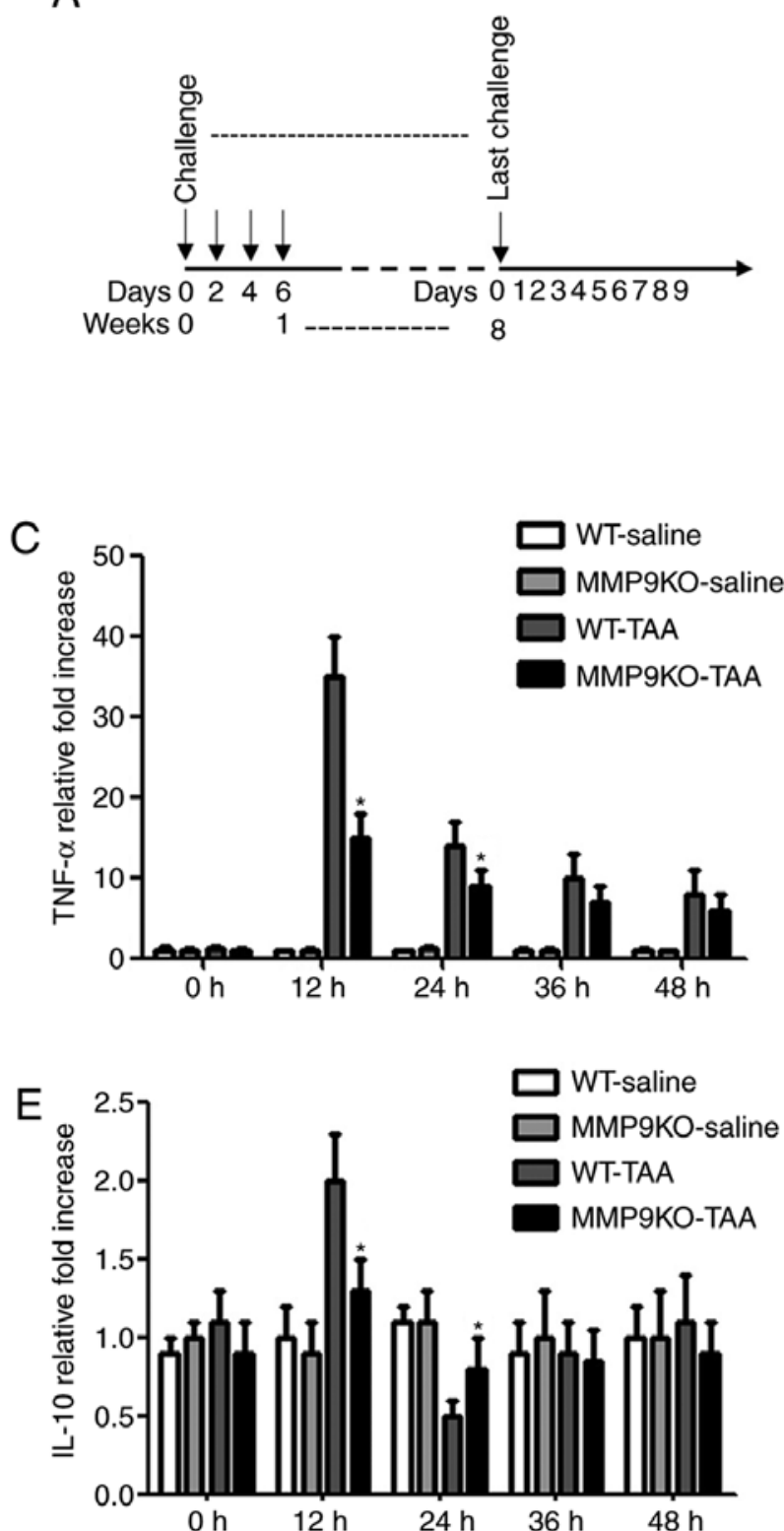
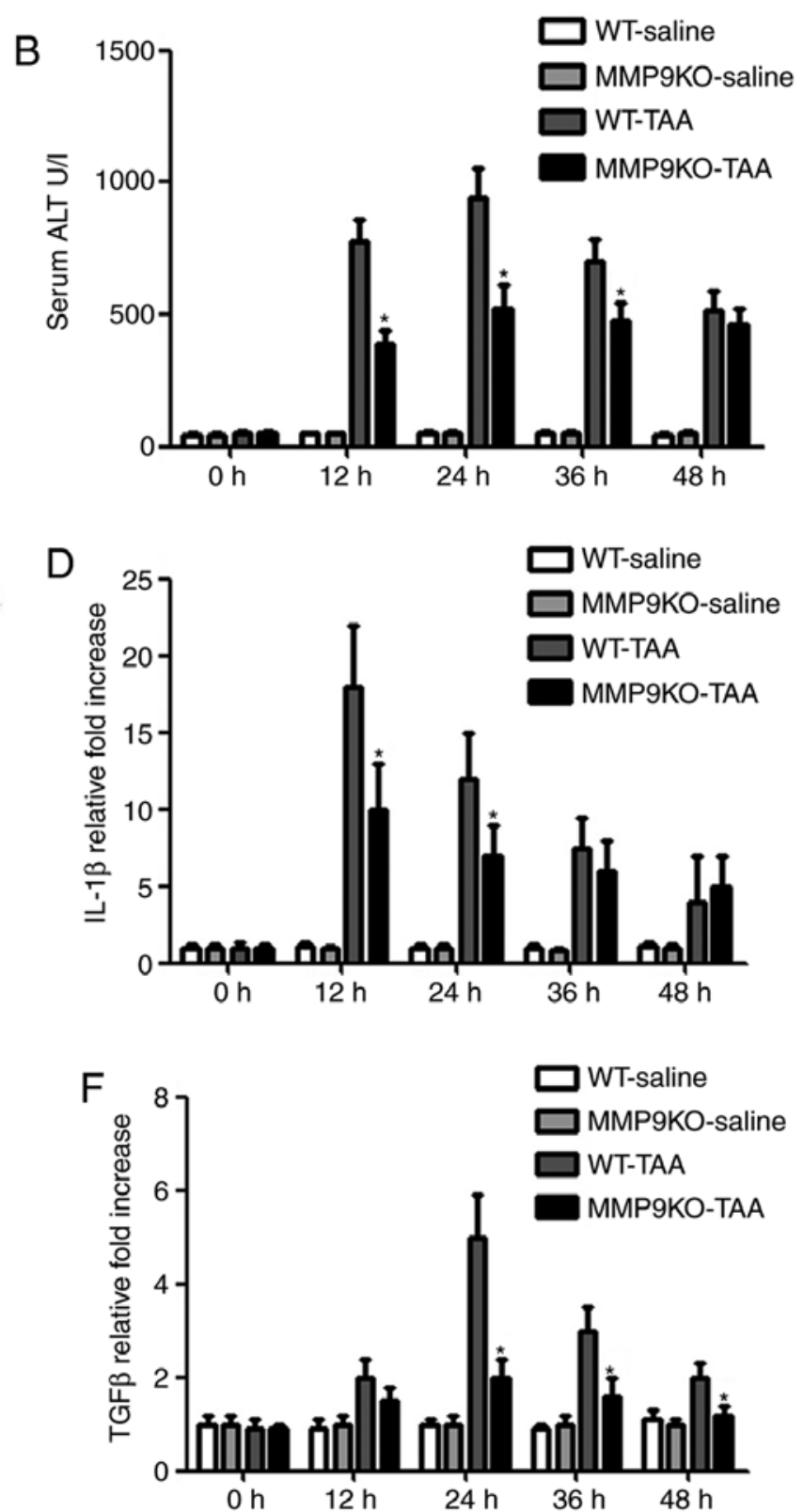

Figure 1. Attenuation of liver injury by depleting MMP9. (A) WT and MMP9- mice were treated with TAA (0.1 mg/g body weight; intraperitoneally) every 2 days for 8 weeks, following which the TAA challenge was withdrawn and fibrosis resolution was observed for 9 days. (B) Serum was collected at the indicated time points. ALT level was measured in a clinical biochemical laboratory. The levels of (C) TNF- $\alpha$, (D) IL-1 $\beta$, (E) IL-10 and (F) TGF $\beta$ in the liver homogenate were determined using commercial ELISA kits. All samples and standards were measured in duplicate. Data are expressed as fold-change compared with the control. "P<0.05 vs. WT animals. MMP9, matrix metalloproteinase-9; WT, wild-type mice; MMP9KO, MMP9-deficient mice; ALT, alanine aminotransferase; TAA, thioacetamide; TNF- $\alpha$, tumor necrosis factor $\alpha$; IL, interleukin; TGF $\beta$, transforming growth factor $\beta$.

Certain animals were sacrificed just prior to the second challenge with TAA in order to analyze the degree of acute liver injury. The serum level of ALT was increased markedly in the WT animals $24 \mathrm{~h}$ after TAA injection, whereas the elevation was less marked in the MMP9 ${ }^{-/}$mice (Fig. 1B). The same variation was observed in the levels of TNF- $\alpha$ and IL-1 $\beta$ (Fig. 1C and D). The level of IL-10 was increased by $98 \%$ at $12 \mathrm{~h}$ and decreased by $48 \%$ at $24 \mathrm{~h}$ in the WT mice compared with that in the saline-treated animals. However, no significant change was observed in the $\mathrm{MMP}^{-/-}$animals (Fig. 1E). The level of TGF $\beta$ was increased 5-fold in the WT animals 2-fold in the $\mathrm{MMP9}^{-/}$animals $24 \mathrm{~h}$ after TAA injection, compared with that in the controls (Fig. 1F).
Liver fibrogenesis occurs alongside the development of liver injury (2). Increasing deposition of collagen types I and III is the paramount feature of liver fibrogenesis $(22,23)$. In the present study, enhanced expression levels of collagens I and III were observed in both genotypic mice following TAA treatment (Fig. 2). However, the quantified data revealed that the depletion of MMP9 decreased the level of collagen I by $55 \%$ at day 1 , and by $72 \%$ at day 2 , compared with that in the WT mice. Collagen III exhibited the same variation in the absence of MMP9 (Fig. 2). Collagen IV is a substrate of MMP9 and also contributes to liver fibrogenesis, although it only presents along vessels or around myofibroblasts (23-25). In the present study, the expression of collagen IV was significantly 


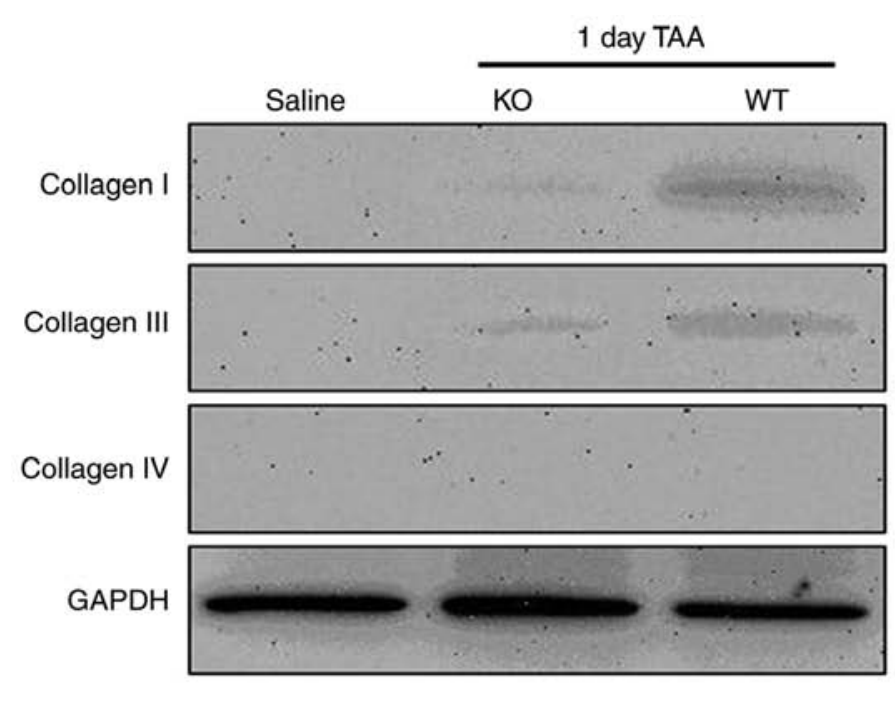

1 day

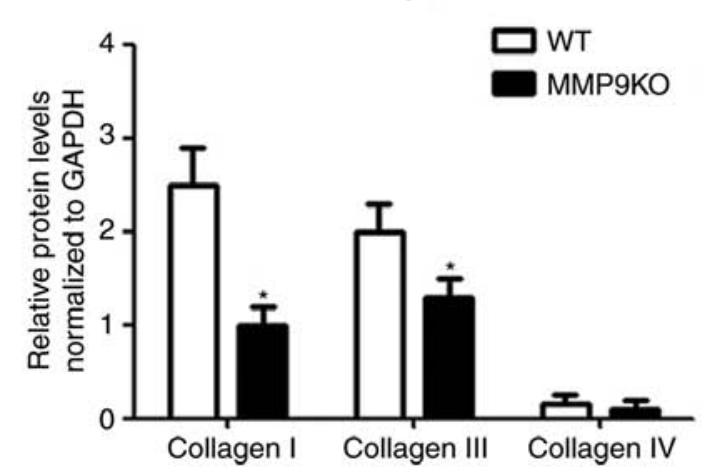

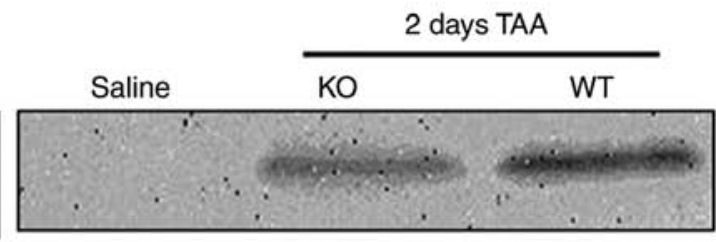
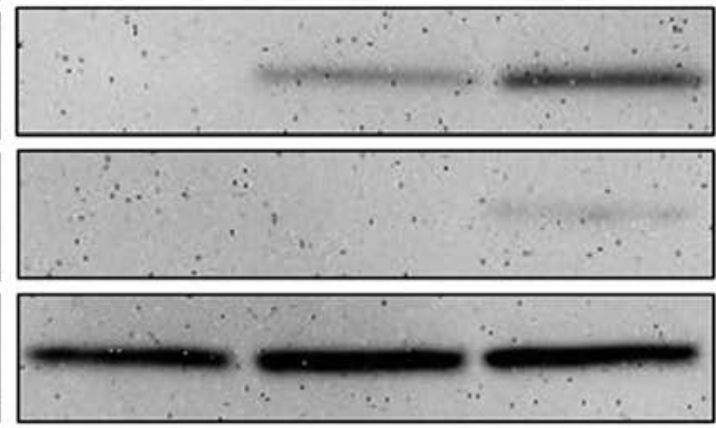

2 days

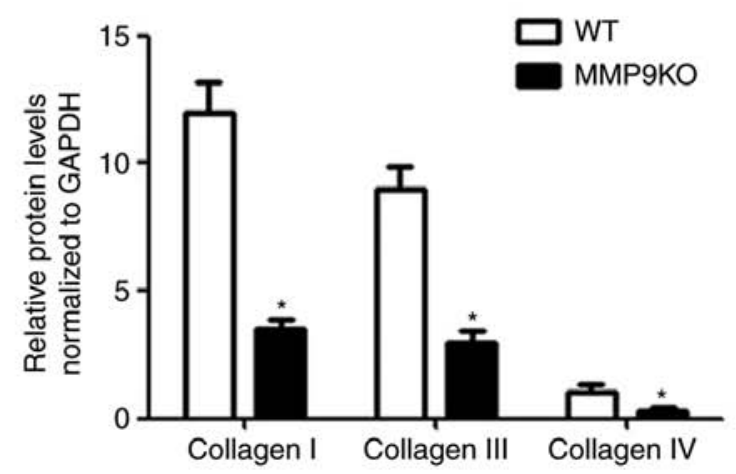

Figure 2. Role of MMP9 in the early stage of fibrogenesis. Protein was extracted from MMP9 ${ }^{-/}$and WT mice 1 or 2 days following TAA treatment. Saline-treated animals were used as the control. The levels of collagen type I, III and IV in liver tissues were determined via western blotting. Total protein concentrations were determined. Data were normalized to GAPDH and expressed as fold-change compared with the control. $\mathrm{n}=5$, " $\mathrm{P}<0.05$ vs. WT animals. MMP9, matrix metalloproteinase-9; WT, wild-type mice; MMP9KO, MMP9-deficient mice; TAA, thioacetamide.

decreased in the absence of MMP9 2 days after TAA treatment, compared with that in the control (Fig. 2).

Dynamic features of collagen deposition and degradation in the absence of MMP9. With continual TAA challenge, the levels of collagens increased with time in both genotypic animals. Compared with that in the WT animals, collagen deposition was slower in the MMP9-/- mice at the early stage of liver fibrosis, particularly during the first 3 weeks. The quantified data demonstrated that the absence of MMP9 decreased collagen I by $46 \%$ at week 1, 57\% at week 3, 49\% at week 5 and $32 \%$ at week 7 (Fig. 3). The expression of collagen III decreased by $33 \%$ at week $1,51 \%$ at week 3 and $37 \%$ at week 5, but there was no significant change at week 7 . The expression of collagen IV decreased by $46 \%$ at week $1,57 \%$ at week $3,49 \%$ at week 5 and $32 \%$ at week 7 (Fig. 3).

The resolution of reversible fibrosis is initiated through the cessation of insults causing liver injury (6). The effect of MMP9 on liver fibrosis resolution was observed for 9 days following the withdrawal of TAA. By quantifying the data from the western blotting, it was revealed that there was no significant difference in the expression of collagen between the two genotypic animals after 8 weeks of TAA treatment when measured on the first day of TAA withdrawal. The levels of collagens decreased faster in the WT animals (Fig. 4). The quantified data revealed that the expression of collagen I decreased by $29 \%$ at day 5 and $58 \%$ at day 9; collagen III decreased by $33 \%$ at day 5 and $69 \%$ at day 9; collagen IV decreased by $21 \%$ at day 5; and $48 \%$ at day 9 , compared with levels in the MMP9-depleted controls (Fig. 4). The histological examination indicated that the resolution of fibrosis was suppressed by the depletion of MMP9 (Fig. 5).

Regulatory effect on the ratio of MMPs/TIMPs by MMP9. Liver injury, wound healing and the activation of MMPs occur in the process of fibrogenesis or fibrosis resolution $(2,13)$. In the present study, the dynamic features of these factors were also demonstrated, which may influence the role of MMP9 in liver fibrosis, however, the underlying molecular mechanism by which MMP9 promotes fibrogenesis or fibrosis resolution requires further investigation. It was observed that the absence of MMP9 decreased the serum level of ALT at the acute injury phase, and modulated the fluctuation of serum ALT at the chronic injury period, although there was no significant difference in the average level of serum ALT compared with that in the controls (Fig. 6). 

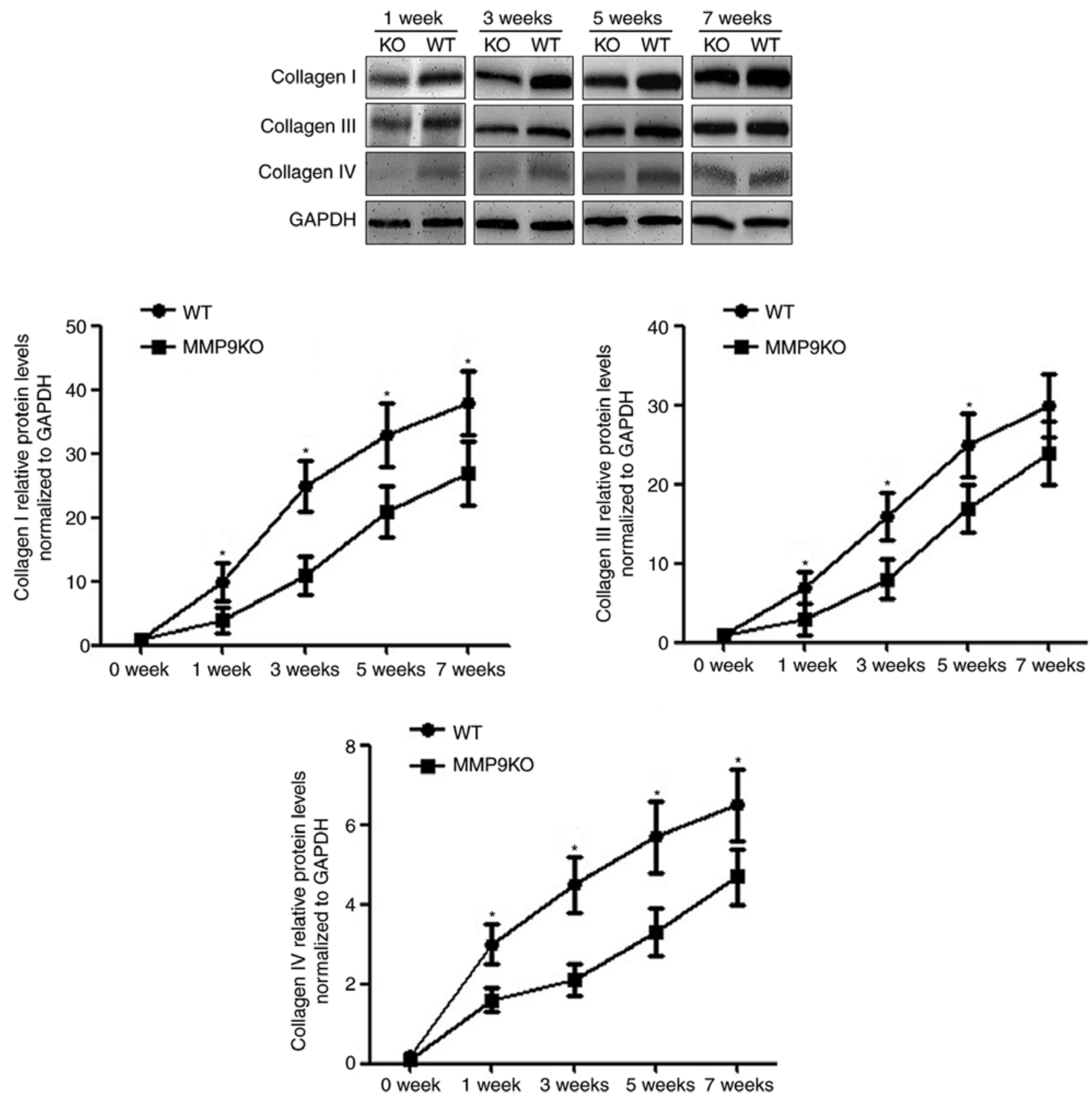

Figure 3. Role of MMP9 in the dynamic process of collagen accumulation. TAA challenge was administered every 2 days for 8 weeks. The protein was extracted from MMP9 ${ }^{--}$and WT mice at weeks $1,3,5$ and 7 . The levels of collagen types I, III and IV in liver tissues were determined via western blotting. To reduce the degradation of protein, western blotting was performed on the day of sample collection. Data were normalized to GAPDH and expressed as the fold-change compared with the control. $n=3$. ${ }^{*} \mathrm{P}<0.05$ vs. WT animals. MMP9, matrix metalloproteinase-9; WT, wild-type mice; MMP9KO, MMP9-deficient mice.

During the development of fibrosis, the mRNA levels of MMP9 (Fig. 7A), MMP13 (Fig. 7B), MMP2 (Fig. 7C) and MMP14 (Fig. 7D) were marginally increased, but there was no significant difference between the two genotypic animals. During the resolution of fibrosis, the mRNA levels of MMPs were significantly decreased. The absence of MMP9 significantly attenuated the decrease in the mRNA level of MMP13 (Fig. 7B).

It was observed that the mRNA levels of TIMP1 (Fig. 8A) and TIMP2 (Fig. 8B) also increased slightly during the first 5 weeks of TAA treatment. The absence of MMP9 decreased the mRNA level of TIMP1, rather than that of TIMP2. During the resolution of fibrosis, the mRNA levels of TIMP1 and
TIMP2 were markedly decreased in the WT animals, but only marginally in the $\mathrm{MMP}^{-/}$mice (Fig. 8A and B).

The actual proteolytic activation of MMPs is dependent on the ratio of MMPs with their corresponding TIMPs. In the present study, it was observed that the absence of MMP9 decreased the (MMP9 + MMP13)/TIMP1 ratio (Fig. 9A). The (MMP2 + MMP14)/TIMP2 ratio was slightly increased during the development of fibrosis and decreased on fibrosis resolution (Fig. 9B). It was also observed that the absence of MMP9 decreased the hepatic level of TGF $\beta$ in the development of fibrosis, whereas its expression was enhanced during fibrosis resolution, particularly notable IX days following TAA withdrawal (Fig. 9C). 

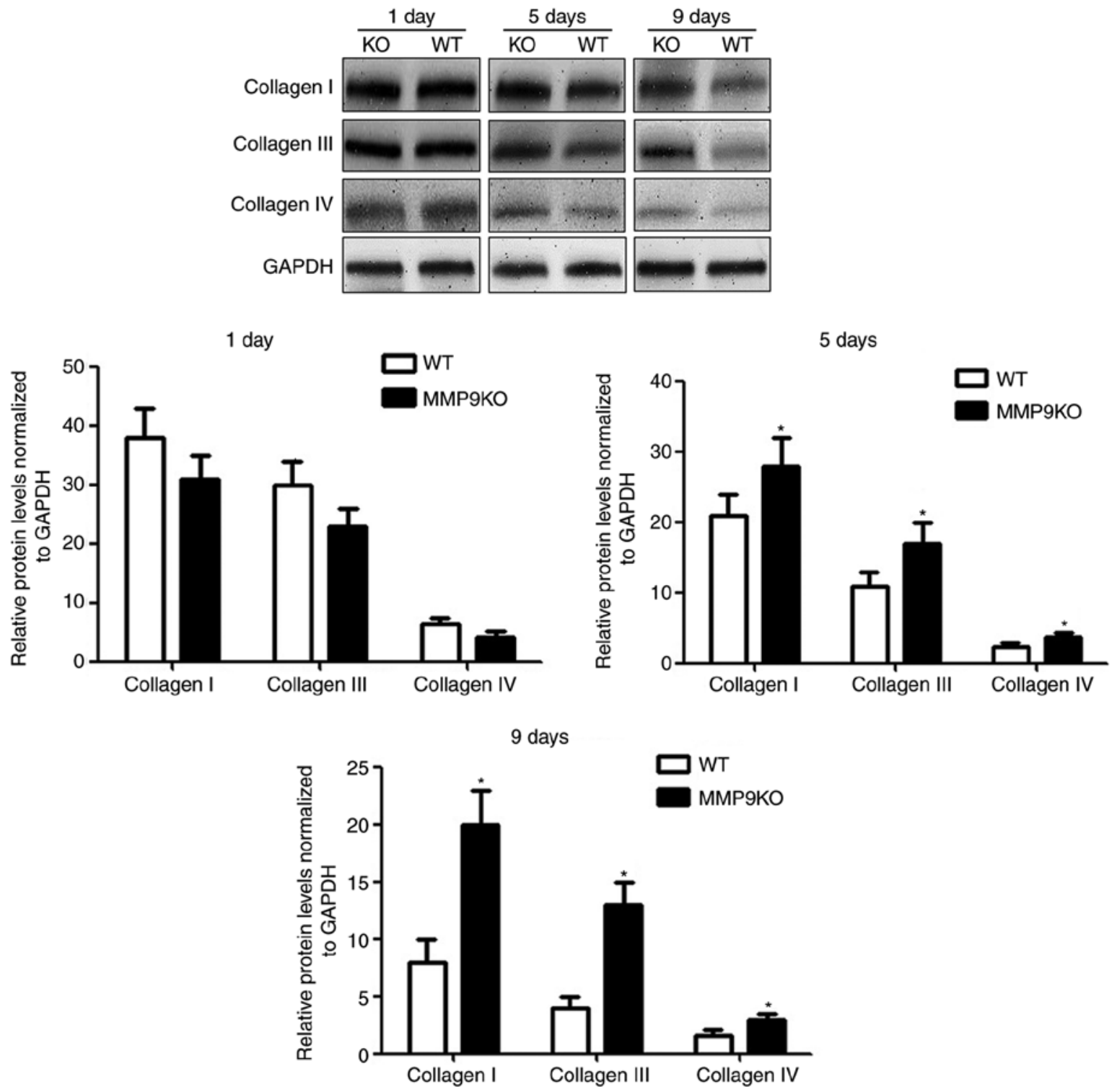

Figure 4. Effect of MMP9 on liver fibrosis resolution. Following TAA withdrawal, protein was extracted from MMP9 ${ }^{-/}$and WT mice on days 1,5 and 9 . The levels of collagen types I, III and IV in liver tissues were determined via western blotting. To reduce degradation of protein, western blotting was performed on the day of sample collection. Data were normalized to GAPDH and expressed as the fold-change compared with the control. $n=5$. ${ }^{*} \mathrm{P}<0.05$ vs. WT animals MMP9, matrix metalloproteinase-9; WT, wild-type mice; MMP9KO, MMP9-deficient mice.

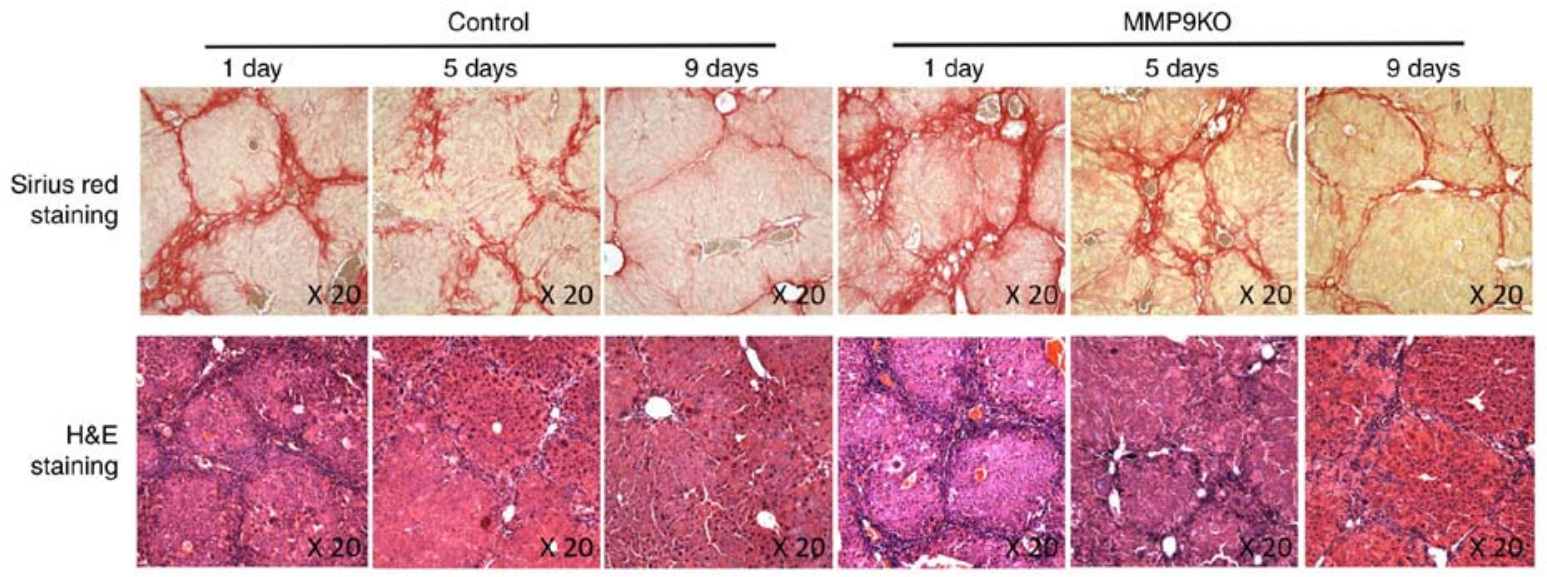

Figure 5. Histological examination of fibrosis resolution in the mice with or without MMP9 depletion. Following TAA withdrawal, the protein was extracted from MMP9 ${ }^{-/}$and WT mice on days 1, 5 and 9. The liver tissue was collected and fixed in $4 \%$ formalin and subsequently embedded in paraffin. Sections were cut into 5-mm slices for subsequent H\&E and Sirius red staining. The results represent three independent tests. WT, wild-type mice; MMP9KO, MMP9-deficient mice; H\&E, hematoxylin and eosin. 


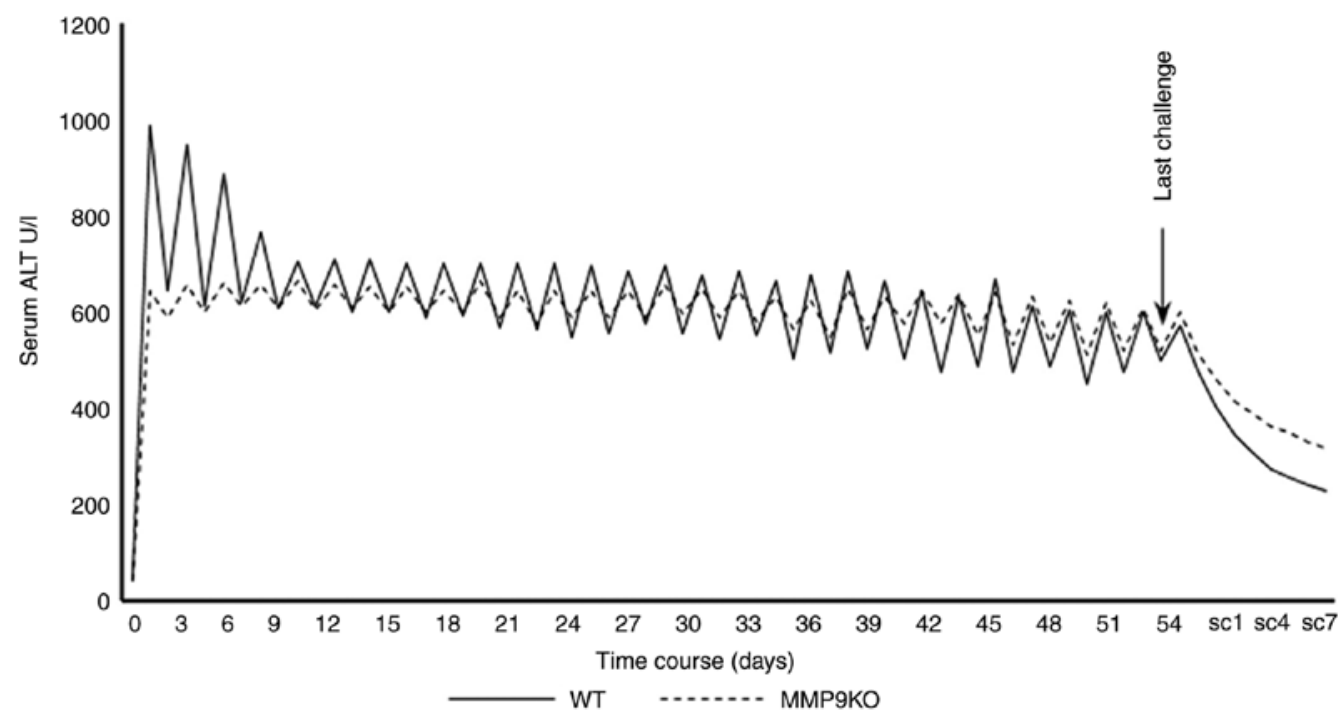

Figure 6. Effect of MMP9 on liver injury and wound healing. Serum was collected via ocular blood extraction at the indicated time points. ALT levels were measured in a clinical biochemical laboratory. Data are expressed as the mean $(\mathrm{n}=3)$. MMP9, matrix metalloproteinase-9; ALT, alanine aminotransferase; WT, wild-type mice; MMP9KO, MMP9-deficient mice.
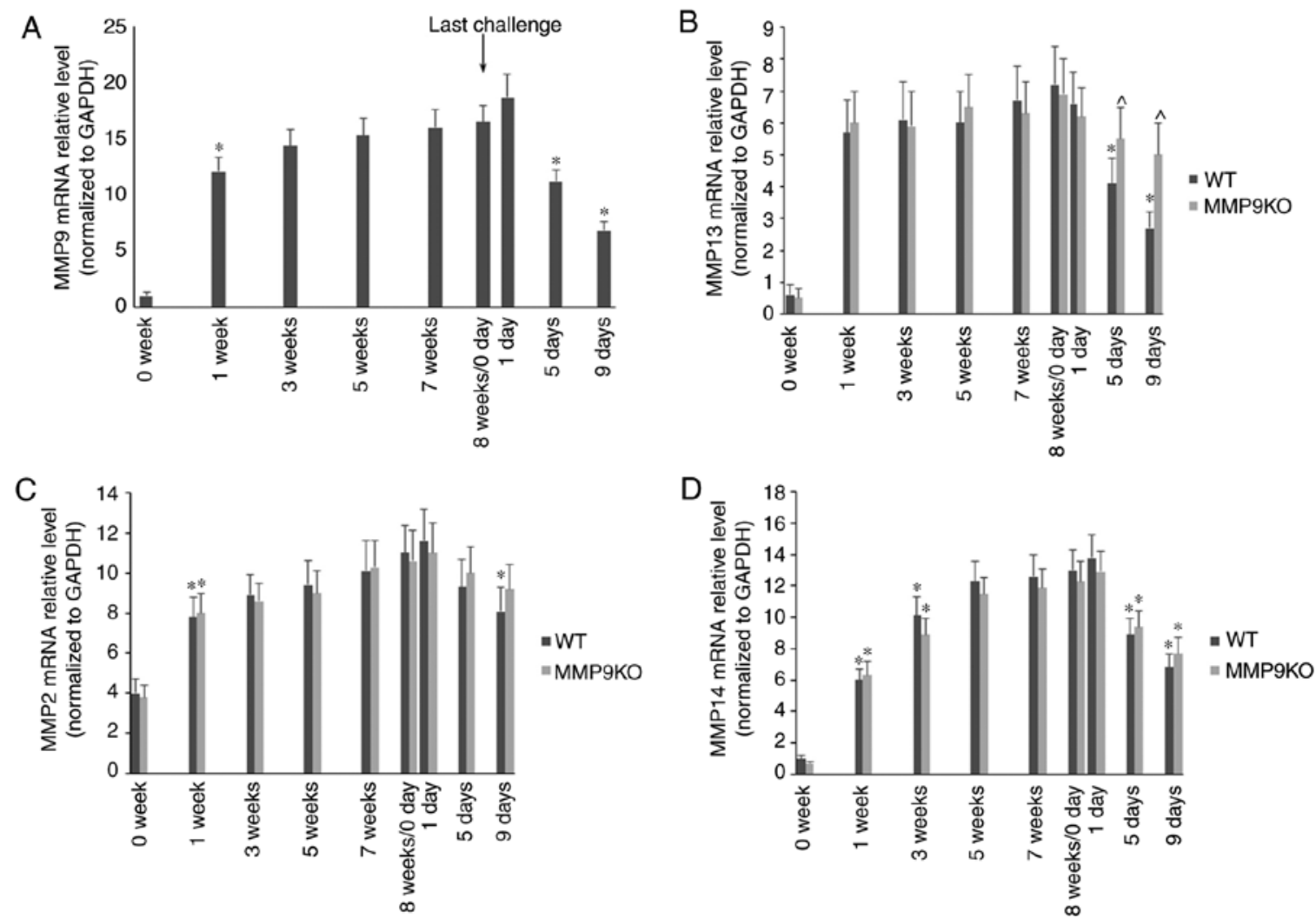

Figure 7. Effect of MMP9 on the transcription of MMPs. Certain MMP9-- and WT mice were sacrificed at the indicated time points. Total mRNA was extracted from the liver of these mice. The levels of (A) MMP9, (B) MMP13, (C) MMP2 and (D) MMP14 were analyzed via reverse transcription-quantitative PCR analysis. Data were normalized to GAPDH and expressed as the fold-change compared with the control. $\mathrm{n}=5 .{ }^{*} \mathrm{P}<0.05$ vs. week $8 ;{ }^{\wedge} \mathrm{P}<0.05$ vs. WT animals. MMP, matrix metalloproteinase; WT, wild-type mice; MMP9KO, MMP9-deficient mice; w, week; d, day.

\section{Discussion}

Despite MMP9 being important in liver fibrogenesis and fibrosis resolution, the dynamic features of liver fibrosis induced by the two-edged effect of MMP9 remain unclear. The present study used the previously established liver fibrotic model of
TAA-induced liver injury and investigated the dynamic features of fibrosis development and regression in the absence of MMP9.

Liver fibrogenesis is driven by inflammation, therefore, inflammatory cytokines in acute liver injury were first determined during the present study. In line with previous studies on ischemia and reperfusion and hepatic toxin-induced liver 

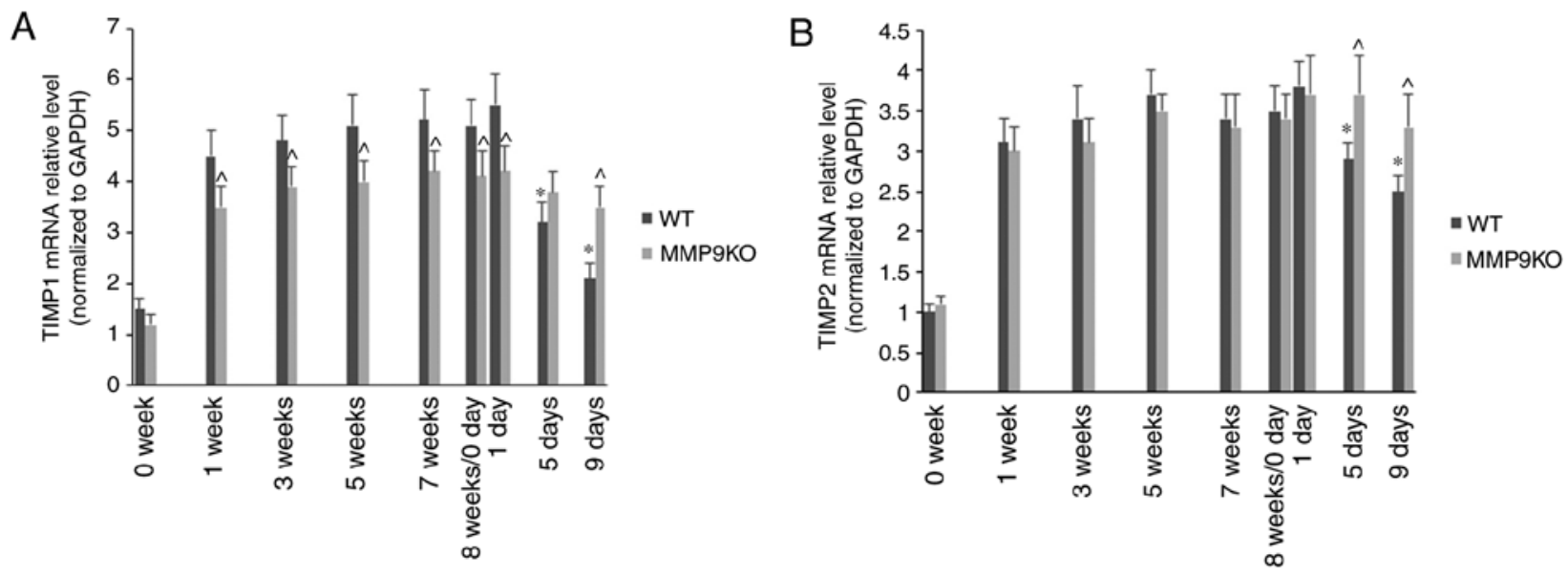

Figure 8. Effect of MMP9 on transcription of TIMPs. Total mRNA was extracted from the liver at the indicated time points. The levels of (A) TIMP1 and (B) TIMP2 were analyzed via reverse transcription-quantitative PCR analysis. Data were normalized to GAPDH and expressed as the fold-change compared with the control. $\mathrm{n}=5$. ${ }^{*} \mathrm{P}<0.05$ vs. week 8 ; ${ }^{\wedge} \mathrm{P}<0.05$ vs. WT animals. MMP9, matrix metalloproteinase-9; TIMP, tissue inhibitor of metalloproteinase; WT, wild-type mice; MMP9KO, MMP9-deficient mice.

A

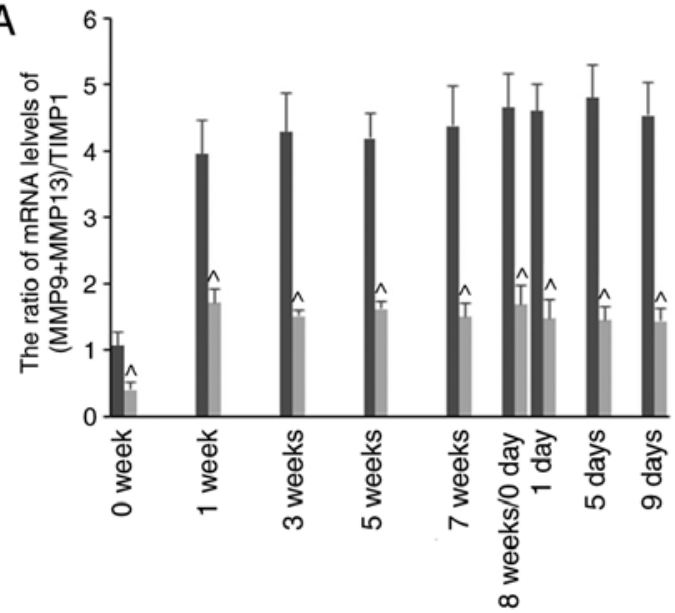

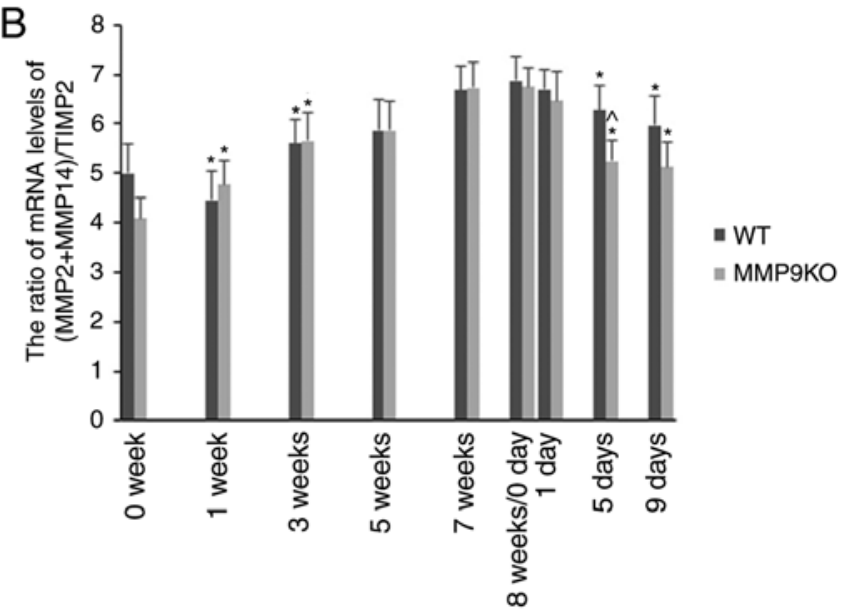

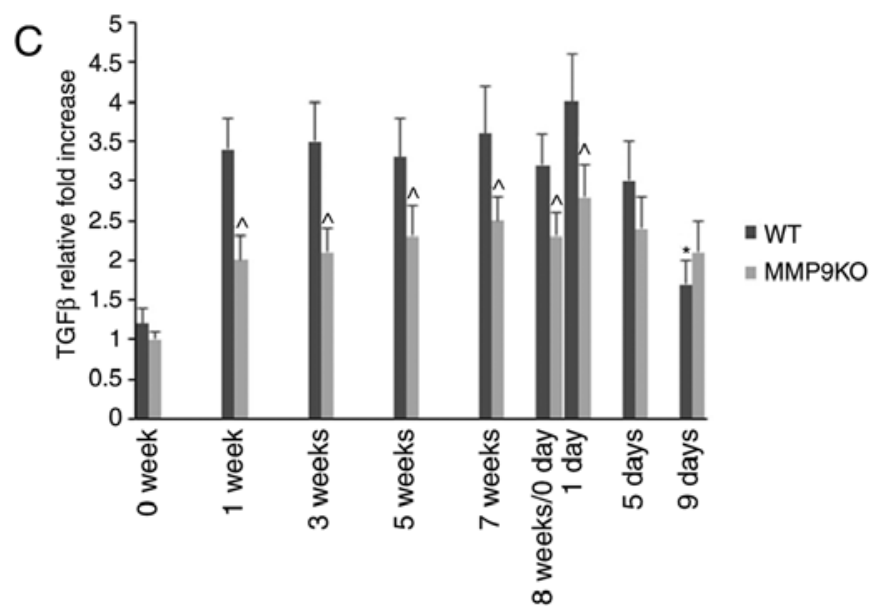

Figure 9. Regulatory effect of MMP9 on MMP/TIMP ratios and the expression of TGF $\beta$. The ratios of (A) (MMP9 + MMP13)/TIMP1 and (B) $($ MMP2 + MMP14)/TIMP2 were calculated based on the mRNA levels of MMP and TIMPs. (C) TGF $\beta$ in the liver homogenate was determined using commercial ELISA kits. Data are expressed as the fold-change compared with the control. $n=5$. ${ }^{\prime} \mathrm{P}<0.05$ vs. week $8 ;{ }^{\wedge} \mathrm{P}<0.05$ vs. WT animals. MMP, matrix metalloproteinase; TIMP, tissue inhibitor of metalloproteinase; TGF $\beta$, transforming growth factor $\beta$; WT, wild-type mice; MMP9KO, MMP9-deficient mice; w, week; d, day.

injury, the depletion of MMP9 attenuated acute liver injury and decreased liver fibrogenesis in the present study (14). The continual TAA challenge utilized in the present study revealed for the first time, to the best of our knowledge, that the depletion of MMP9 attenuated collagen deposition at the early stage of liver fibrosis, although the deposition remained increased 
with time. At the end of the challenge course, the expression of these collagens reached the same levels as those in the control. $\alpha$-smooth muscle actin ( $\alpha$-SMA) is a marker for active HSCs. The depletion of MMP9 suppresses the activation of HSCs and decreases the expression of $\alpha$-SMA (14). Why the long-term effect of MMP9 is not obvious requires further investigation in the future. However, to the best of our knowledge, MMP9 is only one of the MMPs regulating liver fibrogenesis and fibrosis resolution. In the absence of MMP9, other MMPs may serve an important role (10-14).

The resolution of fibrosis is initiated when the cause of cirrhosis is removed. The liver adapts to a near-normal structure (26). In the present study, the absence of MMP9 increased collagen levels. However, the underlying mechanism by which MMP9 regulates the resolution of liver fibrosis remains to be fully elucidated. Our previous study demonstrated that the adoptive transfer of MMP9-expressing kupffer cells promoted fibrosis resolution (20).

Increasing evidence has demonstrated that MMP9 promotes fibrogenesis by activating latent TGF $\beta$, which stimulates the activation of HSCs (10-12). However, the underlying molecular mechanism by which MMP9 facilitates the regression of fibrosis requires further investigation. In general, the process of fibrosis resolution consists of three steps: The transformation of immunoreaction phenotypes following insult withdrawal, particularly the generation of restorative macrophages; the decline in activated HSCs; and the degradation of ECM (6). MMP8 has been demonstrated to promote transformation of the macrophage phenotype (18), whereas MMP9 has been demonstrated to induce the apoptosis of activated HSCs (16). Kupffer-derived MMP9, MMP12 or MMP13 serve critical roles in ECM degradation (6,27). Previous studies have revealed that MMP9 indirectly promoted the resolution of fibrosis by promoting the expression of vascular endothelial growth factor to facilitate transformation to the macrophage phenotype $(3,28)$ and decreasing collagen I to promote the apoptosis of activated HSCs $(16,29)$.

In the present study, the dynamic features of liver injury, wound healing and MMP activation were demonstrated, which are associated with liver fibrosis and its regression (3). It was observed that the absence of MMP9 attenuated acute, rather than chronic, liver injury. Previous studies have demonstrated that MMP9 caused collapse of the hepatic sinus and worsened acute liver injury (30), however, why the absence of MMP9 has no effect on chronic injury remains to be elucidated.

The actual activation of MMPs is dependent on their expression levels and the levels of TIMPs $(4,31)$. In the present study, it was observed that the mRNA levels of MMPs (MMP2, MMP9, MMP13 and MMP14) and TIMPs (TIMP1 and TIMP2) were increased with TAA challenge and were decreased following TAA withdrawal. The (MMP9 + MMP13)/TIMP1 and (MMP2 + MMP14)/TIMP2 ratios were calculated and it was revealed that both ratios were lower in the absence of MMP9. In addition, it was revealed that the absence of MMP9 increased the expression of TGF $\beta$ during the late stage of fibrosis resolution compared with that in the control. The effect of the loss of MMP9 on liver fibrosis can be partially explained by the features of these promotive factors for fibrogenesis or fibrosis resolution, although the precise underlying molecular mechanism requires further investigation.
In conclusion, the present study demonstrated the dynamic features of liver fibrogenesis and fibrosis resolution in the absence of MMP9. These results indicate that the two-edged effect of MMP9 is regulated by the time and state of liver injury. An improved understanding of the role of MMP9 in liver fibrosis will provide a stepping stone for novel clinical applications by targeting MMP9 in the future.

\section{Acknowledgements}

Not applicable.

\section{Funding}

The present study was supported by the National Nature Science Foundation of China (grant nos. 81670561 and 81300336), the Nature Science Foundation of Jiangsu Province (grant nos. QNRC2016022 and 2014-WSW-046), the Nature Science Foundation of Nanjing (grant no. JQX14003) and the Fundamental Research Funds for the Central Universities (grant no. 021414380445).

\section{Availability of data and materials}

All data generated or analyzed during this study are included in this published article.

\section{Authors' contributions}

QW and LL contributed to the conception and design. MF and JW made important modifications to conception and design. QW, XL and JZ made substantial contributions to acquisition and analysis of data. QW interpreted data with help from MF and JW. QW, MF and JW were involved in drafting the manuscript and/or its critical revision for important intellectual content. MF and JW provided final approval of the version to be published. All authors agree to be accountable for all aspects of the work in ensuring that questions related to the accuracy or integrity of any part of the work are appropriately investigated and resolved.

\section{Ethics approval and consent to participate}

The protocol of the present study was approved by the Institutional Animal Care and Research Advisory Committee of Nanjing Drum Tower Hospital (Nanjing, China).

\section{Patient consent for publication}

Not applicable.

\section{Competing interests}

The authors declare that they have no competing interests.

\section{References}

1. Campana L and Iredale JP: Regression of liver fibrosis. Semin Liver Dis 37: 1-10, 2017.

2. Lo RC and Kim H: Histopathological evaluation of liver fibrosis and cirrhosis regression. Clin Mol Hepatol 23: 302-307, 2017. 
3. Ramachandran P, Iredale JP and Fallowfield JA: Resolution of liver fibrosis: Basic mechanisms and clinical relevance. Semin Liver Dis 35: 119-131, 2015.

4. Zhang X, Feng M, Liu X, Bai L, Kong M, Chen Y, Zheng S, Liu S, Wan YJ, Duan Z and Han YP: Persistence of cirrhosis is maintained by intrahepatic regulatory $\mathrm{T}$ cells that inhibit fibrosis resolution by regulating the balance of tissue inhibitors of metalloproteinases and matrix metalloproteinases. Transl Res 169: 67-79.e1-e2, 2016.

5. Altamirano-Barrera A, Barranco-Fragoso B and MéndezSánchez N: Management strategies for liver fibrosis. Ann Hepatol 16: 48-56, 2017.

6. Tacke $\mathrm{F}$ and Trautwein $\mathrm{C}$ : Mechanisms of liver fibrosis resolution. J Hepatol 63: 1038-1039, 2015.

7. George J, Tsutsumi M and Tsuchishima M: MMP-13 deletion decreases profibrogenic molecules and attenuates $\mathrm{N}$-nitrosodimethylamine-induced liver injury and fibrosis in mice. J Cell Mol Med 21: 3821-3835, 2017.

8. Koyama $\mathrm{Y}$ and Brenner DA: Liver inflammation and fibrosis. J Clin Invest 127: 55-64, 2017

9. Han YP: Matrix metalloproteinases, the pros and cons, in liver fibrosis. J Gastroenterol Hepatol 21 (Suppl 3): S88-S91, 2006.

10. Krantz SB, Shields MA, Dangi-Garimella S, Cheon EC, Barron MR, Hwang RF, Rao MS, Grippo PJ, Bentrem DJ and Munshi HG: MT1-MMP cooperates with Kras(G12D) to promote pancreatic fibrosis through increased TGF- $\beta$ signaling. Mol Cancer Res 9: 1294-1304, 2011.

11. Kobayashi T, Kim H, Liu X, Sugiura H, Kohyama T, Fang Q, Wen FQ, Abe S, Wang X, Atkinson JJ, et al: Matrix metalloproteinase- 9 activates TGF- $\beta$ and stimulates fibroblast contraction of collagen gels. Am J Physiol Lung Cell Mol Physiol 306: L1006-L1015, 2014.

12. Dayer $C$ and Stamenkovic I: Recruitment of matrix metalloproteinase-9 (MMP-9) to the fibroblast cell surface by lysyl hydroxylase 3 (LH3) triggers transforming growth factor- $\beta$ (TGF- $\beta$ ) activation and fibroblast differentiation. J Biol Chem 290: 13763-13778, 2015.

13. Feng M, Wang H, Wang Q and Guan W: Matrix metalloprotease 9 promotes liver recovery from ischemia and reperfusion injury. J Surg Res 180: 156-161, 2013

14. Lu L, Feng M, Gu J, Xia Z, Zhang H, Zheng S, Duan Z, Hu R, Wang J, Shi W, et al: Restoration of intrahepatic regulatory $\mathrm{T}$ cells through MMP-9/13-dependent activation of TGF- $\beta$ is critical for immune homeostasis following acute liver injury. J Mol Cell Biol 5: 369-379, 2013.

15. Zhou X, Hovell CJ, Pawley S, Hutchings MI, Arthur MJ, Iredale JP and Benyon RC: Expression of matrix metalloproteinase- 2 and -14 persists during early resolution of experimental liver fibrosis and might contribute to fibrolysis. Liver Int 24 492-501, 2004.

16. Atta H, El-Rehany M, Hammam O, Abdel-Ghany H, Ramzy M, Roderfeld M, Roeb E, Al-Hendy A, Raheim SA, Allam $\mathrm{H}$ and Marey H: Mutant MMP-9 and HGF gene transfer enhance resolution of CCl4-induced liver fibrosis in rats: Role of ASH1 and EZH2 methyltransferases repression. PLoS One 9: e112384, 2014

17. Murphy FR, Issa R, Zhou X, Ratnarajah S, Nagase H, Arthur MJ, Benyon C and Iredale JP: Inhibition of apoptosis of activated hepatic stellate cells by tissue inhibitor of metalloproteinase-1 is mediated via effects on matrix metalloproteinase inhibition: Implications for reversibility of liver fibrosis. J Biol Chem 277: $11069-11076,2002$
18. Wen G, Zhang C, Chen Q, Luong le A, Mustafa A, Ye S and Xiao Q: A novel role of matrix metalloproteinase-8 in macrophage differentiation and polarization. J Biol Chem 290: 19158-19172, 2015

19. Modol T, Brice N, Ruiz de Galarreta M, García Garzón A, Iraburu MJ, Martínez-Irujo JJ and López-Zabalza MJ: Fibronectin peptides as potential regulators of hepatic fibrosis through apoptosis of hepatic stellate cells. J Cell Physiol 230: 546-553, 2015.

20. Feng M, Ding J, Wang M, Zhang J, Zhu X and Guan W: Kupffer-derived matrix metalloproteinase- 9 contributes to liver fibrosis resolution. Int J Biol Sci 14: 1033-1040, 2018.

21. Livak KJ and Schmittgen TD: Analysis of relative gene expression data using real-time quantitative PCR and the 2(-Delta Delta C(T)) method. Methods 25: 402-408, 2001.

22. Karsdal MA, Henriksen K, Nielsen MJ, Byrjalsen I, Leeming DJ, Gardner S, Goodman Z, Patel K, Krag A, Christiansen C and Schuppan D: Fibrogenesis assessed by serological type III collagen formation identifies patients with progressive liver fibrosis and responders to a potential antifibrotic therapy. Am J Physiol Gastrointest Liver Physiol 311: G1009-G1017, 2016.

23. Nieto N: A systems biology approach for understanding the collagen regulatory network in alcoholic liver disease. Liver Int 32: 189-198, 2012.

24. Mak KM, Chu E, Lau KH and Kwong AJ: Liver fibrosis in elderly cadavers: Localization of collagen types I, III, and IV, $\alpha$-smooth muscle actin, and elastic fibers. Anat Rec (Hoboken) 295: 1159-1167, 2012

25. Liu SB, Ikenaga N, Peng ZW, Sverdlov DY, Greenstein A, Smith V, Schuppan D and Popov Y: Lysyl oxidase activity contributes to collagen stabilization during liver fibrosis progression and limits spontaneous fibrosis reversal in mice. FASEB J 30: 1599-1609, 2016.

26. Di Vinicius I, Baptista AP, Barbosa AA and Andrade ZA: Morphological signs of cirrhosis regression. Experimental observations on carbon tetrachloride-induced liver cirrhosis of rats. Pathol Res Pract 201: 449-456, 2005.

27. Ramachandran P, Pellicoro A, Vernon MA, Boulter L, Aucott RL, Ali A, Hartland SN, Snowdon VK, Cappon A, Gordon-Walker TT, et al: Differential Ly-6C expression identifies the recruited macrophage phenotype, which orchestrates the regression of murine liver fibrosis. Proc Natl Acad Sci USA 109: E3186-E3195, 2012.

28. Yamamoto N, Otsuka T, Kondo A, Matsushima-Nishiwaki R, Kuroyanagi G, Kozawa $\mathrm{O}$ and Tokuda $\mathrm{H}$ : Rac limits TGF- $\beta$-induced VEGF synthesis in osteoblasts. Mol Cell Endocrinol 405: 35-41, 2015.

29. Zhu L, Ni B, Liu J, Yang J, Guo Q and Zhou W: Hydroxycamptothecin liposomes inhibit collagen secretion and induce fibroblast apoptosis in a postlaminectomy rabbit model. Eur J Orthop Surg Traumatol 23 (Suppl 1): S85-S91, 2013.

30. Syed I, Rathod J, Parmar M, Corcoran GB and Ray SD: Matrix metalloproteinase-9, -10, and -12, MDM2 and p53 expression in mouse liver during dimethylnitrosamine-induced oxidative stress and genomic injury. Mol Cell Biochem 365: 351-361, 2012

31. Wang JC: Importance of plasma matrix metalloproteinases (MMP) and tissue inhibitors of metalloproteinase (TIMP) in development of fibrosis in agnogenic myeloid metaplasia. Leuk Lymphoma 46: 1261-1268, 2005. 